\title{
Piscine orthoreovirus (PRV) replicates in Atlantic salmon (Salmo salar L.) erythrocytes ex vivo
}

\author{
Øystein Wessel ${ }^{1 *}$, Christel Moræus Olsen ${ }^{1}$, Espen Rimstad ${ }^{1}$ and Maria Krudtaa Dahle ${ }^{2}$
}

\begin{abstract}
Piscine orthoreovirus (PRV) is a reovirus that has predominantly been detected in Atlantic salmon (Salmo salar L.). PRV is associated with heart and skeletal muscle inflammation (HSMI) in farmed Atlantic salmon, and recently erythrocytes were identified as major target cells. The study of PRV replication and pathogenesis of the infection has been impeded by the inability to propagate PRV in vitro. In this study we developed an ex vivo cultivation system for PRV in Atlantic salmon erythrocytes. PRV was successfully passaged to naïve erythrocytes using lysates of blood cells from infected salmon. During cultivation a significant increase in viral load was observed by RT-qPCR and flow cytometry, which coincided with the formation of cytoplasmic inclusions. The inclusions resembled viral factories and contained both PRV protein and dsRNA. In addition, the erythrocytes generated an antiviral immune gene activation after PRV infection, with significant up-regulation of IFN-a, RIG-I, Mx and PKR transcripts. Supernatants from the first passage successfully transmitted virus to naïve erythrocytes. This study demonstrates that PRV replicates in Atlantic salmon erythrocytes ex vivo. The ex vivo infection model closely reflects the situation in vivo and can be used to study the infection and replication mechanisms of PRV, as well as the antiviral immune responses of salmonid erythrocytes.
\end{abstract}

\section{Introduction}

Piscine orthoreovirus (PRV) has a double-stranded RNA (dsRNA) genome and belongs to the family Reoviridae [1]. PRV primarily infects Atlantic salmon (Salmo salar L.) and is associated with heart and skeletal muscle inflammation (HSMI), an important disease of farmed Atlantic salmon in Norway [1,2]. The virus is ubiquitously present in both diseased and apparently healthy individuals [3]. PRV is widespread in farmed salmonids in Europe, North and South America and present in several species of wild salmonids [4-6]. However, PRV has only been associated with disease in farmed Atlantic salmon. The study of viral replication and pathogenesis of the infection has been hindered by the inability to propagate PRV efficiently in cell cultures.

Phylogenetically PRV branches off the common root of the genera Orthoreovirus and Aquareovirus, but clusters more closely with the orthoreoviruses, although it is a fish virus [7-9]. Among orthoreoviruses, mammalian orthoreovirus (MRV) has been studied extensively and

\footnotetext{
* Correspondence: oyfi@nmbu.no

${ }^{1}$ Department of Food Safety and Infection Biology, Norwegian University of Life Sciences, Oslo, Norway

Full list of author information is available at the end of the article
}

used as a model system for investigations of virus-host interactions, which has generated a wealth of information regarding replication cycle, protein functions and virion structure [10]. Even if nucleotide and protein sequence identities between the mammalian orthoreoviruses and PRV are low, important structural motifs and key amino acid are conserved in homologous proteins [7,9]. Currently PRV studies have been restricted to bioinformatic analyses $[4,9]$, RT-qPCR screenings, field epidemiology $[3,6]$ or laborious and costly in vivo challenge studies $[11,12]$. There have been a number of attempts to culture PRV in vitro. Cytopathic effects (CPE) have been observed in several cell lines including epithelioma papulosum cyprini (EPC), fathead minnow (FHM) and GF-1 derived from orange-spotted grouper $[11,13]$. However, increase in PRV titer has not been shown in any of these cell lines. An efficient in vitro cultivation system would be a highly desired tool of PRV research.

The association between PRV and HSMI has been strengthened in recent studies. The high viral RNA loads in the heart and the presence of viral antigens in cardiomyocytes correlate with development of the cardiac lesions observed in HSMI [11,14]. However, an explanation for high viral load in both farmed and wild salmon 
without HSMI lesions is lacking [3,6], and in Canada PRV is present without HSMI being diagnosed [5]. This underlines the importance to study the PRV pathogenesis. We have recently demonstrated high viral loads in blood prior to PRV presence in cardiomyocytes and shown that red blood cells (RBC) are major target cells for PRV [12,14]. At peak infection phase more than 50\% PRV positive erythrocytes were detected in individual fish. Despite the massive PRV infection of RBC, clinical anemia is not associated with HSMI or a PRV infection [15]. The study of PRV infection in erythrocytes will be important to understand the pathogenesis of PRV infection and its implications on Atlantic salmon health.

In MRV infected cells, viral-inclusions are present in distinct areas of the cell during replication; these are recognized as viral factories [16]. The cytoplasmic inclusions observed in PRV infected RBC resemble the globular viral factories of MRV type 3 prototype strain Dearing (T3D) [17,18]. The PRV inclusions contain reovirus-like particles as demonstrated by transmission electron microscopy (TEM) [12]. Inclusions and viruslike particles have previously been reported in erythrocytes of salmonid fish, suggesting that such infections are common [19-22]. The most commonly reported is erythrocytic inclusions body syndrome (EIBS) [23]. However, these findings have not been associated with any specific viral agent. The reovirus-like particles and intraerythrocytic inclusions observed in PRV infected RBC may resemble some of those described as EIBS [24-26]. Piscine erythrocytes, in contrast to mammalian erythrocytes, are nucleated cells containing organelles and capable of protein production $[27,28]$. Therefore, piscine erythrocytes are potentially able to support viral replication.

Recent studies have indicated that piscine erythrocytes may have immune functions [29]. Rainbow trout erythrocytes stimulated with Poly(I:C) respond by expression of genes encoding pathogen receptors (including tolllike receptors (TLRs)), interferon alpha (IFN- $\alpha$ ) and immune cell recruiting mediators like CC chemokine (CCL) 4 [30]. Erythrocytes from Atlantic salmon have been shown to respond to infectious salmon anemia virus (ISAV) by production of IFN- $\alpha$ [31]. An important trigger of interferon production is recognition of dsRNA, either in endosomal compartments by the transmembrane receptor TLR3, or in the cytoplasm by RNA helicases like Retinoic-acid inducible gene (RIG)-I and Melanoma differentiation-associated protein (MDA)-5 [32,33]. In addition, cytosolic dsRNA bind dsRNAactivated kinases like PKR and PKZ and induce partial translational shutdown [34,35]. In order to bypass these antiviral responses, dsRNA viruses have developed counter-mechanisms. These include avoiding the exposure of the dsRNA genome by performing transcription and replication within the viral core, masking the viral RNA by dsRNA-binding viral proteins, inhibiting the dsRNA signaling pathway, or by producing nonpolyadenylated viral mRNA which can omit the translational shutdown [36]. All these mechanisms have been demonstrated for MRV [37,38], and it is likely, due to structural similarities, that PRV counteracts antiviral mechanisms in similar ways. Recently, it was shown that PRV protein o3 binds dsRNA in a sequence independent manner, thus sharing this function with MRV o3 [39].

Through in vivo challenge experiments we have recently found that $\mathrm{RBC}$ is an important target cell for PRV infection in salmon [12], and we hypothesized that PRV can replicate in Atlantic salmon erythrocytes cultures ex vivo. In this study, supernatant from lysed blood cells collected from in vivo PRV infected salmon were passaged to RBC isolated from naïve salmon and cultured ex vivo. The viral propagation was monitored by RT-qPCR, flow cytometry, and immunofluorescence microscopy. The ability of the recipient erythrocytes to elicit an innate antiviral response was also assessed. Finally, a supernatant from the first passage was used to passage PRV to naïve RBC.

\section{Materials and methods Blood samples}

Heparinized blood $(0.5 \mathrm{~mL})$ was collected from the caudal vein of six naïve Atlantic salmon (F1-F6). The fish had an average weight of $40 \mathrm{~g}$ and originated from a naïve population kept in a fresh water tank at VESO aquatic research facility (Vikan, Norway). The fish were anesthetized prior to sampling by bath immersion $(2-5 \mathrm{~min})$ in benzocaine chloride $(0.5 \mathrm{~g} / 10 \mathrm{~L}$ water) and euthanized after sampling using benzocaine chloride ( $1 \mathrm{~g} / 5 \mathrm{~L}$ water) for $5 \mathrm{~min}$. The $\mathrm{RBC}$ were isolated from the blood samples as described below and used as recipient erythrocytes in the first passage. Three weeks later heparinized blood samples were collected from six fish (F11-F16) of the same fish population. RBC were isolated and used as recipient erythrocytes in the second passage.

\section{Isolation of naïve $\mathrm{RBC}$}

We diluted the heparinized blood 1:15 in phosphatebuffered saline (PBS) and isolated the RBC using a Percoll gradient as previously described [12]. The cells were counted using Countess (Invitrogen, Eugene, Oregon, USA) and resuspended to a concentration of $3 \times 10^{7}$ cells/mL in Leibovitz's L15 medium (Life Technologies, Carlsbad, CA, USA) supplemented with fetal calf serum (2\%) and gentamicin $(50 \mu \mathrm{g} / \mathrm{mL})$. All recipient $\mathrm{RBCs}$ (first passage F1-F6; second passage F11-F16) were 
confirmed PRV-negative by RT-qPCR and flow cytometry before initiation of the study (described below).

\section{Preparation of inoculums}

The PRV inoculum was prepared from a batch of pooled heparinized blood samples collected 6 weeks post challenge (wpc) from cohabitant fish of a PRV challenge experiment (VESO, Vikan, Norway). We centrifuged the pooled blood sample at $2000 \mathrm{~g}$ for $10 \mathrm{~min}$ at $4{ }^{\circ} \mathrm{C}$, removed the plasma and diluted the blood pellet 1:10 in L15 with gentamicin $(50 \mu \mathrm{g} / \mathrm{mL})$. The diluted blood was then sonicated on ice by $8 \times 10 \mathrm{~s}$ pulses at $25 \mathrm{~Hz}$ with $30 \mathrm{~s}$ rest in between, and the lysed blood was centrifuged at $2000 \mathrm{~g}$ for $5 \mathrm{~min}$ at $4{ }^{\circ} \mathrm{C}$ to remove cellular debris. The resulting supernatant was used as PRV inoculum in the first passage. Similarly, a negative control inoculum was prepared as described above, using heparinized blood collected from naïve fish. The control inoculum was confirmed PRV negative by RT-qPCR. The PRV inoculum used had a Ct-value of 22.3.

\section{Ex vivo setup; $1^{\text {st }}$ passage}

In the first PRV passage, RBC isolated from six naïve fish were inoculated and cultured ex vivo for 21 days. The culture experiment was performed in 24-deepwell microtiter plates with pyramidal bottom (AB Ninolab, Stockholm, Sweden) to allow easy centrifugation and washing of the cells. The plates were closed using Mikro-Flask sandwich cover and clamp system by Duetz (Applikon, Foster City, CA, USA) to minimize evaporation and prevent well to well contamination. Incubation was performed at $15{ }^{\circ} \mathrm{C}$ in an Ecotron incubation shaker (Infors HT, Basel Switzerland) shaken at $225 \mathrm{rpm}$ to ensure that the RBC was kept in a homogenous suspension.

The cultivation setup included four identical PRV infected plates and four control plates that were sequentially harvested at $1,7,14$ and 21 days post infection (dpi). On each plate, RBC isolated from fish F1-F6 was plated at $3 \times 10^{7} \mathrm{RBC}$ per well in $1 \mathrm{~mL}$ medium. The wells were subsequently inoculated with $100 \mu \mathrm{L}$ of either the PRV-infected inoculate or the control inoculate described above. Following $24 \mathrm{~h}$ of incubation the plates were centrifuged at $500 \mathrm{~g}$ for $5 \mathrm{~min}$ at $4{ }^{\circ} \mathrm{C}$ to remove the supernatant and washed in Leibovitz's L15 medium (Life Technologies). Finally, the RBC was resuspended to a final concentration of $10 \times 10^{6} \mathrm{RBC} / \mathrm{mL}$ in $3 \mathrm{~mL}$ Leibovitz's L15 medium (Life Technologies) supplemented with fetal calf serum $(2 \%)$ and gentamicin $(50 \mu \mathrm{g} / \mathrm{mL})$. The $1 \mathrm{dpi}$ samples were sampled immediately after resuspension and the remaining plates were incubated until harvested at 7,14 and $21 \mathrm{dpi}$ respectively. The $0 \mathrm{dpi}$ samples were collected prior to inoculation.
At each time point a sample of $2 \mathrm{~mL}\left(20 \times 10^{6} \mathrm{RBC}\right)$ was collected from each well for RNA isolation and subsequent RT-qPCR analysis to assess the viral load in the $\mathrm{RBC}$ and the supernatant. In addition, the immune response of infected $\mathrm{RBC}$ was assayed. The remaining $1 \mathrm{~mL}\left(10 \times 10^{6} \mathrm{RBC}\right)$ was used for PRV detection by flow cytometry analysis and immunofluorescence/confocal microscopy to study viral inclusions. The number of cells were counted using Countess (Invitrogen) in order to determine the amount of cell lysis. Details for all analyses are described below.

\section{Ex vivo setup; $2^{\text {nd }}$ passage}

In the second PRV passage, RBC isolated from six fish were infected with supernatant from the first passage and cultured ex vivo for 21 days. A pool of supernatant was prepared from the $21 \mathrm{dpi}$ samples of the first passage after centrifugation at $1000 \mathrm{~g}$ for $5 \mathrm{~min}$ at $4{ }^{\circ} \mathrm{C}$ and used as inoculum.

The cultivation setup included two identical PRV infected plates that were sequentially harvested at 14 and 21 dpi. On each plate, RBC isolated from fish F11-F16 was plated at $3 \times 10^{7} \mathrm{RBC}$ per well in $1 \mathrm{~mL}$ media as for the first passage. The cells were subsequently inoculated with $100 \mu \mathrm{L}$ of a 10 -fold dilution of the supernatant from the first passage. The RBC were incubated for $24 \mathrm{~h}$, centrifuged, washed and resuspended in growth medium to a final concentration of $10 \times 10^{6} \mathrm{RBC} / \mathrm{mL}$. The plates were cultured under identical conditions as those of the first passage and harvested at 14 and 21 dpi. The 0 dpi samples had been collected prior to inoculation.

At each time point a sample of $2 \mathrm{~mL}\left(20 \times 10^{6} \mathrm{RBC}\right)$ was collected from each well for RNA isolation and RTqPCR analysis to detect PRV in the RBC fraction and $1 \mathrm{~mL}$ was used for flow cytometry analysis and immunofluorescence microscopy. Details for the analyses are described below.

\section{RNA isolation}

The $2 \times 10^{6}$ cells $(2 \mathrm{~mL})$ harvested from each culture were pelleted at $1000 \mathrm{~g}$ for $5 \mathrm{~min}$ at $4{ }^{\circ} \mathrm{C}$ and total RNA was isolated from the RBC pellet and the supernatant. The RBC pellet was homogenized in Isol-RNA Lysis Reagent (5 PRIME, Hilden, Germany) using $5 \mathrm{~mm}$ steel beads and TissueLyser II (Qiagen) for $2 \times 5 \mathrm{~min}$ at $25 \mathrm{~Hz}$. After addition of chloroform and centrifugation, the aqueous phase was collected and proceeded with automated RNA isolation using RNeasy Mini QIAcube Kit (Qiagen) as described by the manufacturer. For the supernatant samples, a combination of Trizol LS (Invitrogen) and RNeasy Mini spin column (Qiagen) was used. Briefly, $100 \mu \mathrm{L}$ supernatant was mixed and incubated with Trizol LS before adding chloroform, separating the phases by 
centrifugation, and then proceeding with the RNeasy Mini spin column as recommended by the manufacturer, eluting isolated RNA in $50 \mu \mathrm{L}$ RNase-free water. RNA was quantified using a NanoDrop ND-1000 spectrophotometer (Thermo Fisher Scientific, Wilmington, DE, USA).

\section{RT-qPCR; PRV detection}

The Qiagen OneStep kit (Qiagen) was used for RTqPCR. The input was $100 \mathrm{ng}(5 \mu \mathrm{L}$ of $20 \mathrm{ng} / \mathrm{uL})$ total RNA from $\mathrm{RBC}$ per reaction and $5 \mu \mathrm{L}$ purified RNA solution from the cell free samples, i.e. supernatants and inoculums. RT-qPCR targeted PRV segment S1 using the following conditions: $400 \mathrm{nM}$ primer, $300 \mathrm{nM}$ probe, $400 \mathrm{nM}$ dNTPs, $1.26 \mathrm{mM} \mathrm{MgCl} 2,1: 100$ RNase Out (Invitrogen) and $1 \times$ ROX reference dye with the following cycle parameters: $30 \mathrm{~min}$ at $50{ }^{\circ} \mathrm{C}, 15 \mathrm{~min}$ at $94{ }^{\circ} \mathrm{C}$, 35 cycles of $94{ }^{\circ} \mathrm{C} / 15 \mathrm{~s}, 54{ }^{\circ} \mathrm{C} / 30 \mathrm{~s}$ and $72{ }^{\circ} \mathrm{C} / 15 \mathrm{~s}$ in a Mx3005P (Stratagene, La Jolla, CA, USA). The samples were run in duplicate, and a sample was defined as positive if both parallel samples had a $\mathrm{Ct}<35$. Primers and probe are listed in Table 1.

\section{qPCR; Antiviral response}

Reverse transcription was performed using 200 ng RNA per sample, and a mixed input from representative samples was used to prepare a seven point concentration standard curve. The RNA was denaturated at $95{ }^{\circ} \mathrm{C}$ for 5 min prior to cDNA synthesis, using the Quantitect reverse transcription kit with integrated genomic DNA removal (Qiagen). Quantitative PCR (qPCR) was run in duplicate on cDNA corresponding to 10 ng RNA input. The antiviral response gene expression analysis was performed using $2 \times$ Maxima SYBR Green qPCR Master Mix (Thermo Fisher scientific, Waltham, MA, USA) and $500 \mathrm{nM}$ specific primers targeting Atlantic salmon IFN $\alpha$, myxovirus resistance (Mx), RIG-I and PKR respectively.

Table 1 Primers and probe used in this study

\begin{tabular}{lll}
\hline Primer/probes & Primer sequence $\left(\mathbf{5}^{\prime} \boldsymbol{\rightarrow} \mathbf{3}^{\prime}\right)$ & Accession \# \\
\hline O3 S1-659 Fwd & TGCGTCCTGCGTATGGCACC & GU994022 \\
O3 S1-801 Rev & GGCTGGCATGCCCGATAGCA & \\
O3 S1-693 Probe & FAM-ATCACAACGCCTACCT-MGBNFQ & \\
IFNa Fwd & ACTGAAACGCTACTTCAAGAAGTGA & AY216595 \\
IFNa Rev & GCAGATGACGTTTGTCTCTTCCT & \\
Mx Fwd & GATGCTGCACCTCAAGTCCTATTA & BT043721.1 \\
Mx Rev & CACCAGGTAGCGGATCACCAT & \\
RIG-I Fwd & ACGCCTTGAAGAGCTGGATA & FN178459 \\
RIG-I Rev & CTGGCTGGACTTGTGTCCTC & \\
PKR Fwd & CAGGATGCAACACCATCATC & EF523422.1 \\
PKR Rev & GGTCTTGACCGGTGACATCT & \\
\hline
\end{tabular}

The quencher and reporter dye of the probes are in italic. Accession numbers are shown in the right column.
The assays were run 40 cycles of $94{ }^{\circ} \mathrm{C} / 15 \mathrm{~s}, 60{ }^{\circ} \mathrm{C} / 30 \mathrm{~s}$ in a Mx3005P device (Stratagene). Primers used are listed in Table 1.

\section{Flow cytometry}

RBC sampled during the first and second passage were screened for PRV by flow cytometry. The RBC were stained intracellularly using a rabbit polyclonal antibody against PRV putative outer capsid protein o1 (Anti-or1, \#K275) as previously described [12]. The corresponding zero serum (Anti- $\sigma 1$ Zero \#K275) [14] was used as negative control serum. The cells were analyzed on a Gallios Flow Cytometer (Beckman Coulter, Miami, FL, USA) counting 30000 cells per sample. The data were analyzed using the Kaluza software (Becton Dickinson). The forward/side gate was set to adjust for variation in background staining. The arithmetic mean fluorescence intensity (MFI) was calculated for all PRV infected (green) and control (grey) samples at each time point, in addition to the $\triangle$ MFI. The differences were calculated statistically using Wilcoxon matched pairs signed rank test due to the small sample size $(n=6)$.

\section{Immunofluorescence microscopy}

The PRV $\sigma 1$ stained RBC used in flow cytometry were also assayed by immunofluorescence microscopy. The nuclei were stained with Hoechst 33342 (Invitrogen) before they were transferred to Countess chamber slides (Invitrogen). Photographs were taken by an inverted fluorescence microscope (Olympus IX81), at 20x and $40 \times$ magnification.

\section{Confocal microscopy}

Smears of RBC were dried before fixation for $5 \mathrm{~min}$ in ice cold methanol. Slides were rehydrated in PBS and blocked in PBS with 5\% skimmed milk before sequentially staining with a mouse monoclonal antibody against dsRNA (J2; 1:200) (Scicons, Hungary) and rabbit anti-o1 (1:750) with washing in-between. Secondary antibody; goat anti-mouse Alexa Fluor 488 and goat anti-rabbit Alexa Fluor 594 (1:1000) were obtained from Molecular Probes (Invitrogen). The nucleus was counterstained with Hoechst 33342 (Invitrogen) and coverslips were mounted with Fluoroshield (Sigma-Aldrich). The images were generated on an inverted Zeiss LSM 710 confocal laser scanning microscope (CLSM) (Carl Zeiss, Oberkochen, Germany), using lasers of $405 \mathrm{~nm}$, $488 \mathrm{~nm}$ and $594 \mathrm{~nm}$ to excite the respective stains. In post-production, the original color of dsRNA (Alexa Flour 488) and PRV-ơ1 protein (Alexa Flour 594) was switched to match the immunofluorescence pictures, in which PRV-o1 protein was colored green. 


\section{Data analysis}

The PRV RT-qPCR results from 1 dpi were compared to paired samples collected at 7,14 and $21 \mathrm{dpi}$ in the first. For the immune gene analysis of IFN $\alpha, \mathrm{Mx}$, RIG-I and PKR the fold increase at $1,7,14$ and 21 dpi was calculated, assigning the qPCR results from the uninfected paired samples as 1 . In the second passage, the results from the undiluted and the 10 fold diluted inoculum was compared at each time point. The differences were analyzed statistically using Wilcoxon matched pairs signed rank test due to the small sample size $(n=6)$. All statistical analysis described were performed with GraphPad Prism (GraphPad Software inc., USA) and $p$-values of $p \leq 0.05$ were considered as significant.

\section{Results}

A blood cell lysate prepared from PRV infected fish was used as inoculum in the first passage of PRV to naïve erythrocytes. The inoculum had $\mathrm{Ct}$ value of 22.3. The recipient naïve $R B C$ were confirmed negative for PRV by both RT-qPCR and flow cytometry before onset of the study and they were cultured ex vivo for $21 \mathrm{dpi}$.

\section{Increased PRV RNA shown by RT-qPCR}

Following inoculation with PRV containing lysate, there was an increase in the PRV titer in both RBC and supernatant during the first passage (Figure 1). At $1 \mathrm{dpi}$, after removal of the inoculum and washing of the cells, the mean $\mathrm{Ct}$ value was $21.2 \pm 0.6$ in the $\mathrm{RBC}$ fraction. A significant increase in viral load was observed from $1 \mathrm{dpi}$ to $14 \mathrm{dpi}$ when the $\mathrm{Ct}$ was $14.6 \pm 0.9(p<0.05)$, and it stayed on this level until the end of the first passage at 21 dpi (Ct 14.9 \pm 0.7 ). Similarly, in the supernatant there was a significant increase in mean Ct-value from $1 \mathrm{dpi}$ (Ct $33.4 \pm 1.1)$ to $21 \mathrm{dpi}(\mathrm{Ct} 27.0 \pm 1.8)(p<0.05)$.

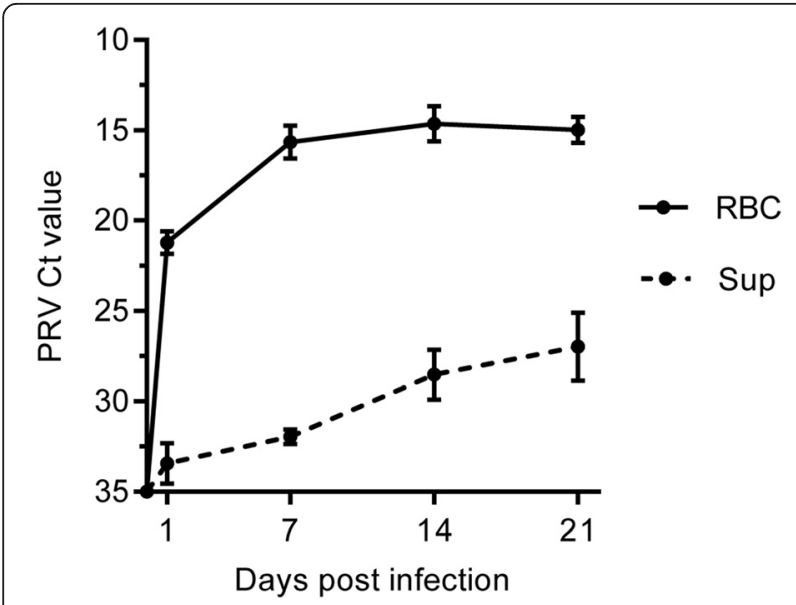

Figure 1 PRV load in $1^{\text {st }}$ passage of culturing. Mean PRV Ct value with SD $(n=6)$ as detected by RT-qPCR in red blood cells (RBC) and supernatant (Sup) at 1, 7, 14 and 21 days post infection.

\section{Increased PRV protein shown by flow cytometry}

The infected $\mathrm{RBC}$ were stained intracellularly with a PRV o1-antibody and analyzed by flow cytometry to determine the level of viral protein during the first passage. At 1 dpi the RBC population observed in the PRVinfected cultures had shifted to the right compared to the uninfected controls (Figure 2A; $1 \mathrm{dpi}$ ), indicating cell association of PRV. At 7 dpi the main population partly overlapped the control culture, but a distinct right shoulder, constituting RBC with higher PRV or1 content, was evident in infected cultures (Figure 2A; 7 dpi). At 14 dpi a broader right shoulder was observed, and the entire PRV infected population had shifted to the right indicating a general increased amount of PRV o 1 protein (Figure 2A; $14 \mathrm{dpi}$ ). At $21 \mathrm{dpi}$ the PRV infected cells were separated into two populations; a PRV high and low positive population (Figure 2A; $21 \mathrm{dpi}$ ).

The pattern described above was observed in all infected cultures and was accompanied by elevated mean fluorescence intensity (MFI) (Figure 2B). The detection of PRV o1, as measured by $\triangle \mathrm{MFI}$, was significantly increased from each time point to the next during the first passage $(p<0.05)$ (Figure $2 \mathrm{C})$. Whereas the main increase in viral load detected by RT-qPCR appeared during the early phases from $1 \mathrm{dpi}$ to $7 \mathrm{dpi}$, the most prominent increase in viral protein was observed in later phases from 7 to $21 \mathrm{dpi}$ (Figure 2D).

\section{Development of erythrocytic PRV inclusions}

PRV infected erythrocytes from passage one were inspected by immunofluorescence (IF) microscopy. At 1 dpi PRV positive staining was observed as small cytoplasmic granules within infected RBC (Figure 3A; 1 dpi). At 7 dpi a similar granular staining pattern was detected, including positive cells with increased fluorescence intensity (Figure 3A; $7 \mathrm{dpi}$ ). At $14 \mathrm{dpi}$ positive cells with prominent large inclusion was observed. (Figure 3A; 14 dpi). The cellular morphology of the PRV infected cells varied, as both elongated ellipsoidal RBC and erythrocytes with rounded cell shape and nucleus was observed. At $21 \mathrm{dpi}$ the number of cells with large inclusions was numerous (Figure 3A; $21 \mathrm{dpi}$ ). The large inclusions were also visible in phase contrast and co-localized with the immunofluorescent staining of PRV. The staining pattern observed by IF, i.e. increasing amounts of PRV o1, was in line with the flow cytometry observations.

We showed that the erythrocytic inclusions contained both dsRNA and PRV o 1 protein by double staining of smears of infected RBC. The dsRNA staining partly or completely co-localized with the PRV or 1 as observed by confocal imaging (Figure 3B). Fixation with methanol was necessary for detection of dsRNA in the co-lolcalization experiments. This fixation technique reduced the signal to noise ratio compared to the method used for 
A

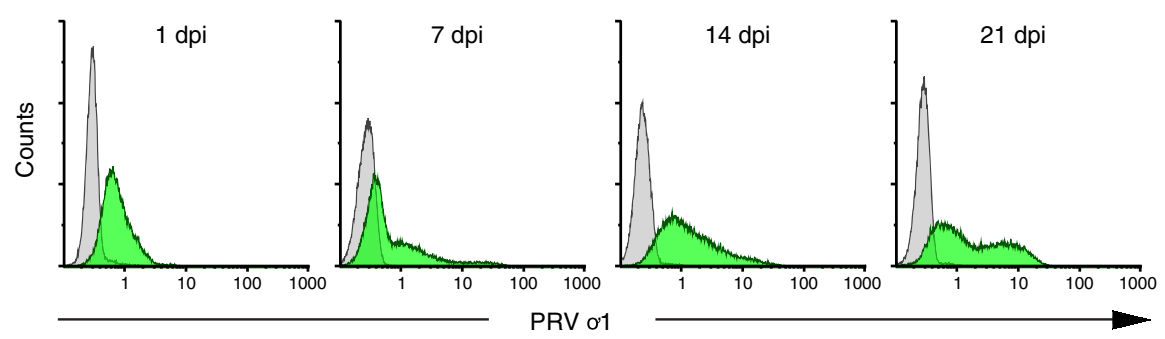

B

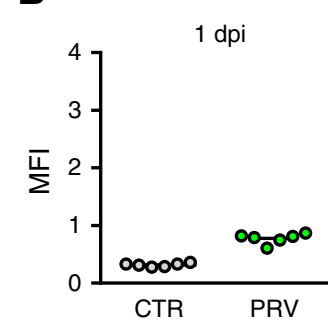

C

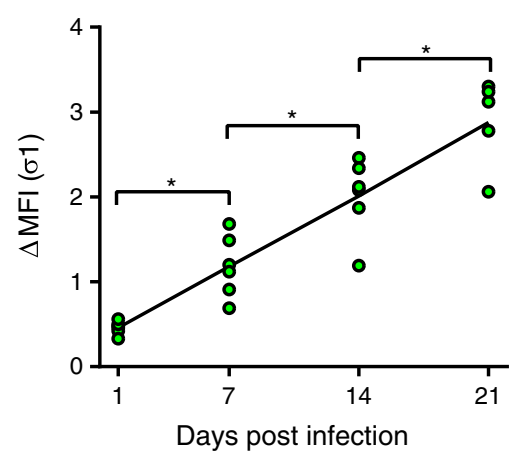

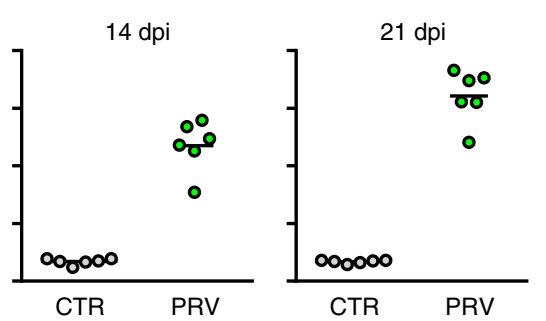

D

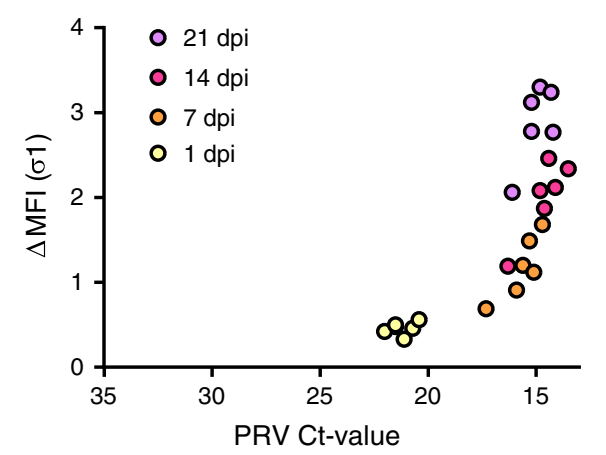

Figure 2 Detection of PRV ơ1 protein by flow cytometry in the $1^{\text {st }}$ passage. (A) Results from intracellular staining for PRV $\sigma^{1}$ protein from PRV infected culture (green) and control culture (grey) at 1, 7, 14 and 21 days post infection (dpi). The fluorescence intensity (PRV $\sigma 1$ ) is shown on the $\mathrm{X}$-axis and cell count on the $\mathrm{y}$-axis counting 30000 cells per sample. The results of one individual are presented to illustrate the staining pattern. (B) Plot of the arithmetic mean fluorescence intensity (MFI) for all PRV infected (green) and control (grey) cultures $(n=6)$ at each time point. (C) The amount of PRV o1 protein detected presented by $\triangle M F I$ (MFI Infected - MFI Control) at 1, 7, 14 and 21 dpi. Data were analyzed using Wilcoxon matched pairs signed rank test. ${ }^{*} p<0.05$. (D) The correlation between the amount of PRV nucleic acid and PRV $\sigma 1$ protein detected during the cultivation is illustrated by plotting PRV Ct-values (X-axis) against the $\triangle M F I$ for PRV $\sigma 1$ (Y-axis) color coding the different time points; 1 dpi (yellow), 7 dpi (orange), 14 dpi (red) and 21 dpi (purple).

flow cytometry and standard immunofluorescence microscopy (Figure 3A).

\section{Ex vivo infected $\mathrm{RBC}$ elicit an antiviral immune response} In order to determine if the PRV infected $R B C$ responded to PRV infection by antiviral gene expression, the mRNA levels of IFN- $\alpha, \mathrm{Mx}$, RIG-I and PKR were analyzed. IFN- $\alpha$ was significantly up-regulated at $1 \mathrm{dpi}$ (Figure 4A). A significant up-regulation at 1 and $7 \mathrm{dpi}$ was observed for Mx (Figure 4B), RIG-I (Figure 4C) and PKR (Figure 4D). These latter three genes followed a similar expression pattern with peak expression detected at $7 \mathrm{dpi}$, before the expression levels returned to baseline.

\section{Successful passage of PRV}

Tenfold dilutions of the supernatant from passage 1 were used as inoculums in passage 2 . The undiluted and 10-fold diluted inoculums were measured to $\mathrm{Ct}$ values of 27.0 and 31.6 respectively. Following inoculation there was an increase in viral load from $1 \mathrm{dpi}$ until $14 \mathrm{dpi}$, and similar to passage 1 the increase leveled off thereafter (Figure 5A). The peak viral load was significantly higher in $\mathrm{RBC}$ given the undiluted compared to the 10 fold diluted inoculum at $14 \mathrm{dpi}$ (Ct $20.5 \pm 0.6$ and Ct $22.9 \pm 0.3$ respectively) and $21 \mathrm{dpi}$ (Ct $21.2 \pm 0.7$ and Ct $23.0 \pm 0.6$ respectively) $(p<0.05)$. In line with passage 1 , PRV o 1 positive RBC were observed at 14 and 21 dpi by IF microscopy (Figure 5B). However, the number of positive 


\section{A}

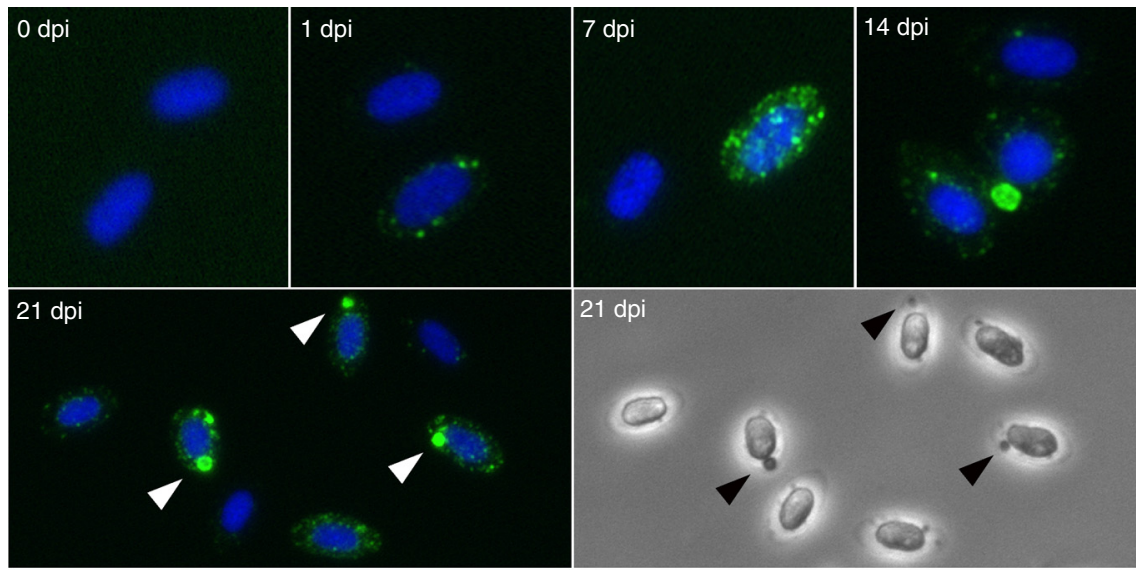

B

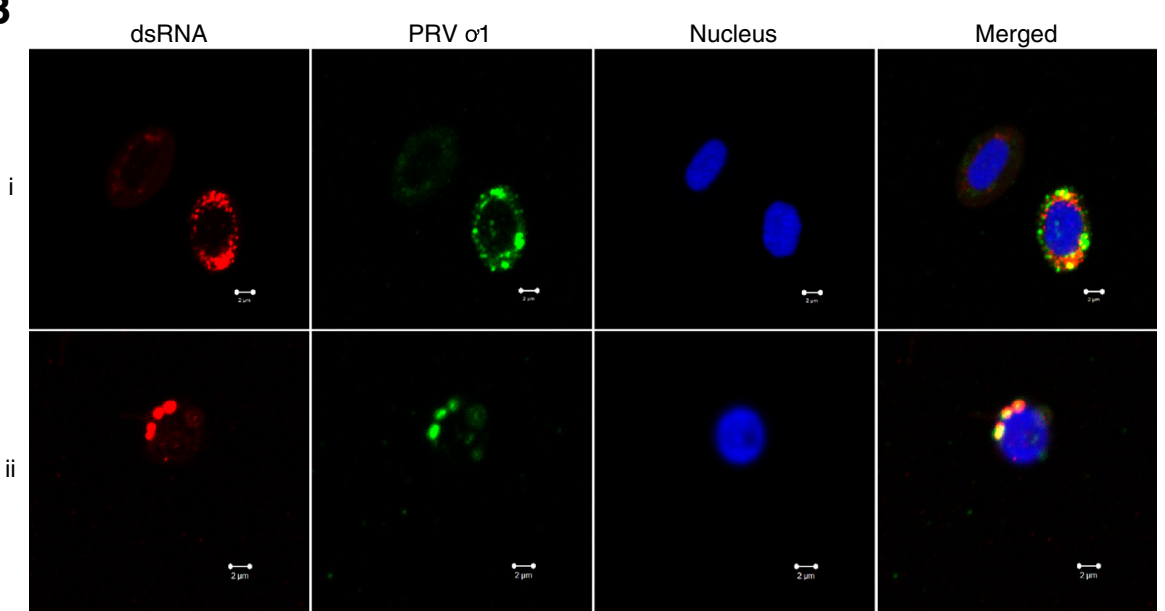

Figure 3 Immunofluorescence and confocal microscopy of PRV infected RBC. (A) Fluorescent labeling of the PRV $\sigma 1$-protein (green) in ex vivo infected RBC. The nuclei were stained with Hoechst (blue). Non-infected cells are shown at 0 dpi, and infected cells are shown at 1, 714 and 21 dpi. Large PRV inclusions at $21 \mathrm{dpi}$ (marked by arrowheads) can also be observed in phase contrast. (B) Confocal images of ex vivo infected RBC at 21 dpi, stained with anti-dsRNA (red) and anti-PRV- $\sigma 1$ (green). The nuclei were stained with Hoechst (blue). dsRNA was detected within PRV inclusions, and either partly (i) or completely (ii) co-localized with PRV $\sigma 1$. Scale bar $2 \mu \mathrm{m}$.

cells was much lower, and the PRV positive population was below the detection level for flow cytometry (data not shown).

\section{Discussion}

In this study we demonstrated that PRV replicates in Atlantic salmon erythrocytes ex vivo. PRV obtained from infected fish were passaged to naïve erythrocytes. A significant increase in viral load was detected and viral factories containing PRV proteins and dsRNA was formed within infected RBC. Supernatant from the first passage was successfully passaged to naive erythrocytes. In addition, we demonstrated that ex vivo infected $\mathrm{RBC}$ generated an innate antiviral immune response.
During the first passage, a significant increase in PRV load in the RBC fraction was observed by RT-qPCR and flow cytometry. At $1 \mathrm{dpi}$ the mean $\mathrm{Ct}$-value was relatively low, indicating that the added PRV efficiently associated with $\mathrm{RBC}$. The results of flow cytometry corroborated this as the entire $\mathrm{RBC}$ population shifted to the right, suggesting that all RBC were PRV positive at this time point. The increase in the amount of viral RNA was most prominent in the early phase and plateaued at $14 \mathrm{dpi}$. The peak $\mathrm{Ct}$-values were similar to those observed in previous in vivo challenge trials [12]. On the other hand, the viral load as detected by flow cytometry increased until $21 \mathrm{dpi}$, indicating a continuous production of PRV proteins within infected RBC. During ex vivo cultivation, PRV infected RBC formed two 
A

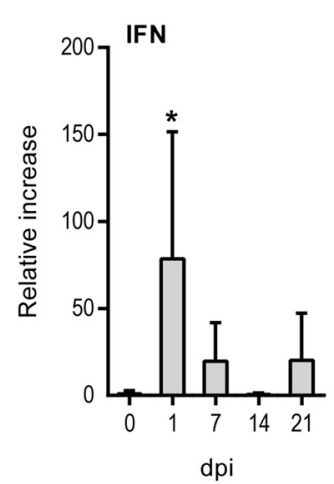

B

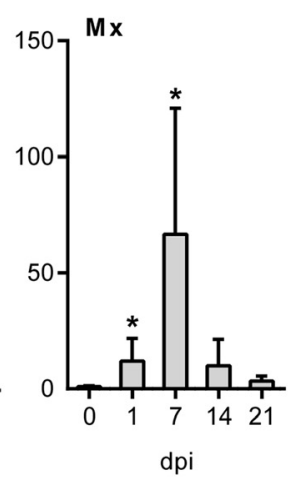

C

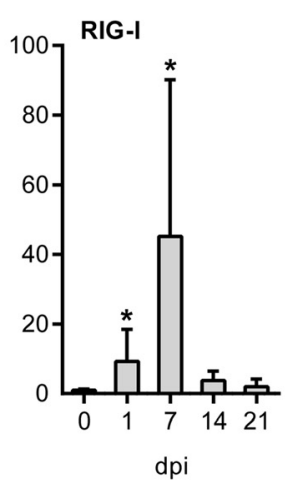

D

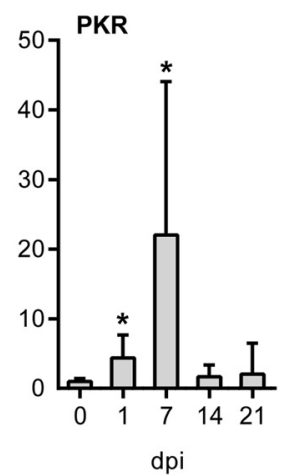

Figure 4 Erythrocytic antiviral responses to PRV infection. Expression of genes involved in antiviral responses was measured by RT-qPCR. The expression levels in infected RBC relative to the paired non-infected controls were calculated for each sample $(n=6)$ at $1,7,12$ and 21 dpi. The relative increase (and SD) for IFNa (A), Mx (B), RIG-I (C) and PKR (D) is shown. Data were analyzed using Wilcoxon matched pairs signed rank test. ${ }^{*} p<0.05$.

distinct populations observed by flow cytometry. This is similar to that observed during in vivo challenge experiments in which low and high PRV positive RBC populations are found during peak PRV infection [12].

The amount of PRV protein detected by flow cytometry were in line with the PRV staining observed by immunofluorescence microscopy. The PRV staining was observed as cytoplasmic inclusions similar to those observed during in vivo studies [12], and developed over time from small numerous granules to large distinct inclusions. At $14 \mathrm{dpi}$ and $21 \mathrm{dpi}$, many RBC with one or two very large inclusions were observed, probably reflecting the high PRV positive RBC population. The inclusions were shown to contain both dsRNA and PRV or protein, although the degree of co-localization varied between cells. For MRV, the viral replication process takes place in cytoplasmic inclusions called viral factories [16]. These viral factories are shown to contain large amounts of both viral proteins and dsRNA and are essential for successful replication [16,40]. The globular inclusions detected in PRV infected RBC resembles the shape of the viral factories observed in MRV type 3 Dearing (T3D) infected cells $[12,17,18]$. Thus, both content and morphology of PRV inclusions are similar to the viral factories of orthoreoviruses.

A significant increase in PRV load was observed in the supernatant, which successfully passaged PRV to naïve erythrocytes. This indicates that infectious viral progeny is produced during the ex vivo cultivation. RBC with PRV inclusions were observed in the second passage,
A

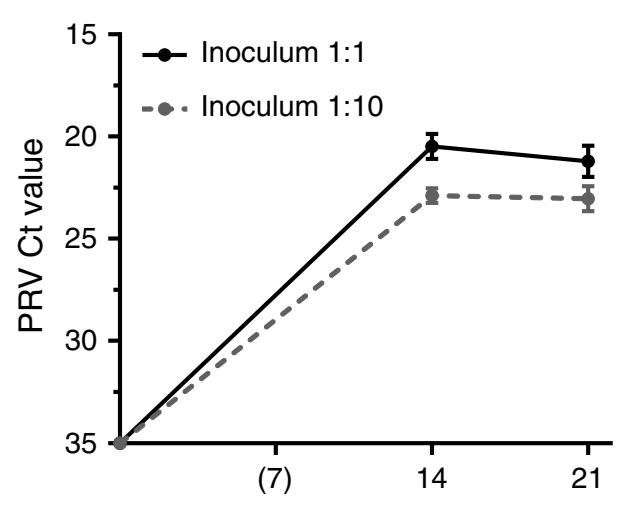

B

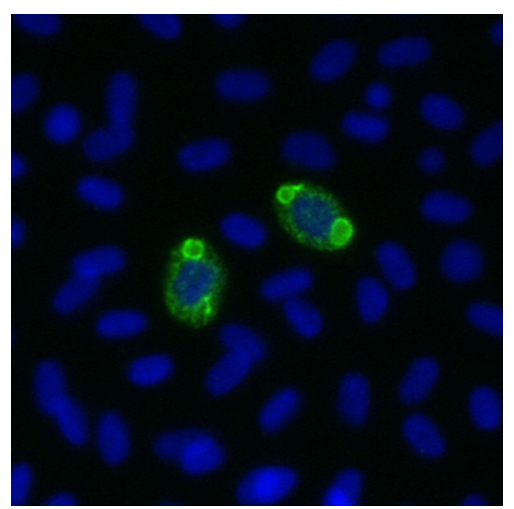

Figure 5 RT-qPCR and PRV ơ 1 staining from the $2^{\text {nd }}$ passage. (A) Mean PRV Ct value (and SD) detected by RT-qPCR in red blood cells (RBC) at 14 and $21 \mathrm{dpi}(n=6)$ during the second passage. Results are shown for cultures infected with undiluted and diluted (1:10) inoculum from the 1st passage. (B) Immunofluorescence microscopy picture showing the PRV $\sigma 1$-protein (green) in cells at 14 dpi infected by undiluted inoculum. Cell nuclei stained with Hoechst (blue). 
however, the number of positive cells was low and below detection level for flow cytometry. In previous in vivo experiments, the threshold of detection by flow cytometry appeared to be corresponding to a Ct-value of about 20 [12], thus slightly below the level obtained in the second passage. The viral titer of the inoculum was significantly higher in passage 1 (Ct-value 22.3) compared to passage 2 (Ct-value 27.0). This could explain the difference in virus outcome between the passages.

The amount of PRV within infected RBC was substantial; however, it was not accompanied by cell lysis (data not shown). The low, but increasing viral load detected in the supernatant suggests a slow but steady release of virus. This correlates well with in vivo observations where neither a PRV infection or an HSMI outbreak is associated with anemia [15]. However, HSMI is associated with circulatory disturbances, and a swollen spleen with high viral load is a common finding [12,41]. Orthoreovirus infections commonly results in cell lysis and release of progeny particles is thought to follow cell death and disruption of the plasma membrane [10]. However, non-lytic persistent infections have also been demonstrated in cell cultures [42-46]. The release mechanism of PRV remains to be shown. Although the present study suggests salmon $\mathrm{RBC}$ can tolerate high amounts of PRV, it is not known how it affects other important erythrocyte functions, such as oxygen transport.

Viral replication in erythrocytes is not well documented in the literature. Mature mammalian erythrocytes are not nucleated and thus not able to support a viral replication [47]. However, it is demonstrated that MRV can bind erythrocytes by the $\sigma 1$ protein $[48,49]$, and amino acid residues involved in this binding are partly conserved in the PRV $\sigma 1$ protein $[7,9]$. In contrast to mammalian RBC, piscine erythrocytes are nucleated and retain ribosomes and a number of organelles while in circulation, thus competent to perform transcription and protein synthesis $[27,30]$. Differences in the content of PRV protein in $\mathrm{RBC}$ were observed during the present ex vivo infection and in previous in vivo experiments, indicating that the susceptibility of RBC varies [12]. During maturation and ageing of erythrocytes there is an inverse relationship between loss of cytoplasmic organelles and increase in hemoglobin content $[50,51]$ accompanied by a reduced aerobic metabolic rate [52]. Both total RNA content and protein synthesis is higher in young erythrocytes [28]. It is a possibility that the putative differences in PRV susceptibility of erythrocytes is correlated to maturation.

PRV is the first virus shown to replicate in salmonid erythrocytes. However, there have been several reports of different viral inclusions in salmonid erythrocytes [19-22]. The most commonly reported is erythrocytic inclusions body syndrome (EIBS) [23], which have been associated with a transient anemia in freshwater Pacific salmon [53]. In contrast, EIBS has not been linked to anemia in Atlantic salmon [54]. No specific viral agent has been characterized for these findings. Interestingly, EM images of PRV and PRV inclusions strongly resemble some of those reported for EIBS [12,24-26]. Blood smears collected from experimentally PRV infected fish have been shown to stain positive by pinacyanol chloride [12]; the most commonly used method in detection and characterization of EIBS. However, a report suggested the EIBS agent to be enveloped, whereas PRV is a reovirus which is non-enveloped [55]. The present study demonstrated that erythrocytic PRV inclusions contain dsRNA, whereas a previous report suggested the EIBS agent to be a single stranded RNA virus based on acridine orange staining [26]. Pinacyanol chlorid and acridine orange are not agent specific staining methods, in contrast to the dsRNA MAb and PRV $\sigma 1$ specific antibody used in the present study. Whether EIBS represents one viral agent or several related or non-related viral agents is not known and should be sorted out.

Prior studies have shown that salmon erythrocytes can elicit antiviral responses to Poly(I:C) stimulation [30]. We observed that PRV infection ex vivo induced gene expression of IFN $\alpha, \mathrm{Mx}$, RIG-I and PKR, indicating the mobilization of an antiviral response. The production of $\mathrm{Mx}$, which is an interferon target gene [56,57], indicate that interferon is also produced at the protein level following PRV infection. Expression of RIG-I and PKR in particular indicate that the induced response may specifically target dsRNA viruses. This response may have the ability to inhibit or moderate further PRV infection and replication in the RBC cultures, leading to suboptimal viral production, and indicating that viral propagation in $\mathrm{RBC}$ ex vivo can be optimized further.

RBC is a major target cell for PRV in vivo and thus the study of PRV infection in erythrocytes will be central to progress our understanding of PRV infection and its implications on Atlantic salmon health. The lack of available cell lines supporting PRV replication has restricted studies of PRV infection and pathogenesis. The present study demonstrated an ex vivo infection model using primary naïve erythrocytes, which could be used to propagate PRV. The advantage of using RBC would be that it closely mimics the in vivo situation; therefore, the ex vivo cultivation model will likely benefit future studies of PRV infection, replication and release from RBC. In addition, it could be used to study the antiviral immune response of erythrocytes in salmonid fish.

\section{Abbreviations}

CCL: CC chemokine ligand; Ct: Cycle threshold; dpi: Days post infection; dsRNA: Double-stranded RNA; EIBS: Erythrocytic inclusion body syndrome; EPC: Epithelioma papulosum cyprinid; FHM: Fathead minnow; HSMI: Heart and skeletal muscle inflammation; IFN-a: Interferon alpha; ISAV: Infectious 
salmon anemia virus; MDA: Melanoma differentiation-associated; MFI: Mean fluorescence intensity; MRV: Mammalian orthoreovirus; MX: Myxovirus resistance; PBS: Phosphate-buffered saline; PRV: Piscine orthoreovirus; RBC: Red blood cells; RIG: Retinoic-acid inducible gene; RT-qPCR: Real-time quantitative polymerase chain reaction; T3D: Type 3 Dearing; TEM: Transmission electron microscopy; TLR: Toll-like receptor

\section{Competing interests}

A patent application for the ex vivo propagation of PRV in RBC has been sent to the Norwegian Patent Office 30.08.2013 as patent application number NO 20131163. This may become public on 28.02.2015. The patent application has also been filed internationally as PCT/EP2014/068293. We have received no reimbursements, fees, funding, or salary linked to this patent application.

\section{Authors' contributions}

$\varnothing W$ participated in the overall design of the study, performed sampling, RBC isolation, RT-qPCR analysis, flow cytometry and immunofluorescence microscopy, interpretation of the data and drafted the manuscript. CMO helped in blood smear staining and confocal microscopy and in interpretation of the overall data. ER participated in the overall design of the study, helped in interpretation of the data and drafting the manuscript. MKD participated in the overall design of the study, GPCR analysis, helped in interpretation of the data, drafting the manuscript and coordination of the study. All authors read and approved the final manuscript.

\section{Acknowledgements}

Thanks to the team at VESO Vikan, including Marie Løvoll and Christian Wallace, for conducting the challenge experiment, and to Randi Faller and Even Thoen at the Norwegian Veterinary Institute for technical assistance and confocal imaging respectively.

\section{Author details}

${ }^{1}$ Department of Food Safety and Infection Biology, Norwegian University of Life Sciences, Oslo, Norway. ${ }^{2}$ Section of Immunology, Norwegian Veterinary Institute, Oslo, Norway.

\section{Received: 14 May 2014 Accepted: 26 January 2015} Published online: 06 March 2015

\section{References}

1. Palacios G, Løvoll M, Tengs T, Hornig M, Hutchison S, Hui J, Kongtorp RT, Savji N, Bussetti AV, Solovyov A, Kristoffersen AB, Celone C, Street C, Trifonov V, Hirschberg DL, Rabadan R, Egholm M, Rimstad E, Lipkin WI (2010) Heart and skeletal muscle inflammation of farmed salmon is associated with infection with a novel reovirus. PLoS One 5:e11487

2. Kongtorp RT, Kjerstad A, Taksdal T, Guttvik A, Falk K (2004) Heart and skeletal muscle inflammation in Atlantic salmon, Salmo salar L.: a new infectious disease. J Fish Dis 27:351-358

3. Løvoll M, Alarcon M, Bang JB, Taksdal T, Kristoffersen AB, Tengs T (2012) Quantification of piscine reovirus (PRV) at different stages of Atlantic salmon Salmo salar production. Dis Aquat Organ 99:7-12

4. Kibenge MJ, Iwamoto T, Wang Y, Morton A, Godoy MG, Kibenge FS (2013) Whole-genome analysis of piscine reovirus (PRV) shows PRV represents a new genus in family Reoviridae and its genome segment $\mathrm{S} 1$ sequences group it into two separate sub-genotypes. Virol J 10:230

5. Marty GD, Morrison DB, Bidulka J, Joseph T, Siah A. Piscine reovirus in wild and farmed salmonids in British Columbia, Canada: 1974-2013. J Fish Dis, in press.

6. Garseth AH, Fritsvold C, Opheim M, Skjerve E, Biering E (2013) Piscine reovirus (PRV) in wild Atlantic salmon, Salmo salar L., and sea-trout, Salmo trutta L., in Norway. J Fish Dis 36:483-493

7. Key T, Read J, Nibert ML, Duncan R (2013) Piscine reovirus encodes a cytotoxic, non-fusogenic, integral membrane protein and previously unrecognized virion outer-capsid proteins. J Gen Virol 94:1039-1050

8. Nibert ML, Duncan R (2013) Bioinformatics of recent aqua- and orthoreovirus isolates from fish: Evolutionary gain or loss of FAST and fiber proteins and taxonomic implications. PLoS One 8:e68607

9. Markussen T, Dahle MK, Tengs T, Løvoll M, Finstad ØW, Wiik-Nielsen CR, Grove S, Lauksund S, Robertsen B, Rimstad E (2013) Sequence analysis of the genome of piscine orthoreovirus (PRV) associated with heart and skeletal muscle inflammation (HSMI) in Atlantic salmon (Salmo salar). PLoS One 8:e70075

10. Schiff LA, Nibert ML, Tyler KL (2007) Orthoreoviruses and their replication. In: Knipe DM, Howley PM, Fields BN (ed) Fields virology, 5th edition, vol 2. Wolters Kluwer/Lippincott Williams \& Wilkins, Philadelphia, pp 1853-1915

11. Mikalsen AB, Haugland $\varnothing$, Rode $M$, Solbakk IT, Evensen $\varnothing$ (2012) Atlantic salmon reovirus infection causes a CD8 T cell myocarditis in Atlantic salmon (Salmo salar L.). PLoS One 7:e37269

12. Finstad OW, Dahle MK, Lindholm TH, Nyman IB, Lovoll M, Wallace C, Olsen CM, Storset AK, Rimstad E (2014) Piscine orthoreovirus (PRV) infects Atlantic salmon erythrocytes. Vet Res 45:35

13. Kongtorp RT, Taksdal T (2009) Studies with experimental transmission of heart and skeletal muscle inflammation in Atlantic salmon, Salmo salar L. J Fish Dis 32:253-262

14. Finstad ØW, Falk K, Løvoll M, Evensen $\varnothing$, Rimstad E (2012) Immunohistochemical detection of piscine reovirus (PRV) in hearts of Atlantic salmon coincide with the course of heart and skeletal muscle inflammation (HSMI). Vet Res 43:27

15. Kongtorp RT, Taksdal T, Lyngøy A (2004) Pathology of heart and skeletal muscle inflammation (HSMI) in farmed Atlantic salmon Salmo salar. Dis Aquat Organ 59:217-224

16. Fields BN, Raine CS, Baum SG (1971) Temperature-sensitive mutants of reovirus type 3: Defects in viral maturation as studied by immunofluorescence and electron microscopy. Virology 43:569-578

17. Broering TJ, Parker JS, Joyce PL, Kim J, Nibert ML (2002) Mammalian reovirus nonstructural protein $\mathrm{NNS}$ forms large inclusions and colocalizes with reovirus microtubule-associated protein $\mu 2$ in transfected cells. J Virol 76:8285-8297

18. Parker JS, Broering TJ, Kim J, Higgins DE, Nibert ML (2002) Reovirus core protein $\mu 2$ determines the filamentous morphology of viral inclusion bodies by interacting with and stabilizing microtubules. J Virol 76:4483-4496

19. Landholt ML, MacMillan JR, Patterson M (1977) Detection of an intraerythrocytic virus in rainbow trout (Salmo gairdneri). Fish Health News 6:4-6

20. Hedrick RP, McDowell T, Groff JM (1987) Another erythrocytic virus from salmonid fish? Am Fish Soc Fish Health Sect Newsl 15:5

21. Thorud KE, Lunder T, Wiik S, Engeland E (1990) Electron microscopic observations of EIBSV-like particles and two intraerythrocytic virus-like particles different from EIBSV in Atlantic salmon, Salmo salar, in Norway. Bull Eur Ass Fish Pathol 104:95-97

22. Evelyn TPT, Traxler GS (1978) Viral erythrocytic necrosis: natural occurrence in Pacific salmon and experimental transmission. J Fish Res Board Can 35:903-907

23. Leek SL (1987) Viral erythrocytic inclusion body syndrome (EIBS) occurring in juvenile spring chinook salmon (Oncorhynchus tshawytscha) reared in freshwater. Can J Fish Aquat Sci 44:685-688

24. Lunder T, Thorud K, Holt RA, Rohovec JS (1990) Particles similar to the virus of erythrocytic inclusion body syndrome, EIBS, detected in Atlantic salmon (Salmo salar) in Norway. Bull Eur Assoc Fish Pathol 10:21-23

25. Michak P, Smith CE, Hopper K (1992) Erythrocytic inclusion body syndrome: a light and electron microscopic study of infected erythrocytes of chinook Oncorhynchus tshawytscha and coho 0. kisutch salmon. Dis Aquat Organ 12:229-233

26. Rodger HD (2007) Erythrocytic inclusion body syndrome virus in wild Atlantic salmon, Salmo salar L. J Fish Dis 30:411-418

27. Claver JA, Quaglia AIE (2009) Comparative morphology, development, and function of blood cells in nonmammalian vertebrates. J Exot Pet Med 18:87-97

28. Lund SG, Phillips MC, Moyes CD, Tufts BL (2000) The effects of cell ageing on protein synthesis in rainbow trout (Oncorhynchus mykiss) red blood cells. J Exp Biol 203:2219-2228

29. Morera D, Mackenzie SA (2011) Is there a direct role for erythrocytes in the immune response? Vet Res 42:89

30. Morera D, Roher N, Ribas L, Balasch JC, Donate C, Callol A, Boltana S, Roberts S, Goetz G, Goetz FW, MacKenzie SA (2011) RNA-Seq reveals an integrated immune response in nucleated erythrocytes. PLoS One 6:e26998

31. Workenhe ST, Kibenge MJ, Wright GM, Wadowska DW, Groman DB, Kibenge FS (2008) Infectious salmon anaemia virus replication and induction of alpha interferon in Atlantic salmon erythrocytes. Virol J 5:36

32. Jacobs BL, Langland JO (1996) When two strands are better than one: the mediators and modulators of the cellular responses to double-stranded RNA. Virology 219:339-349 
33. Saito T, Gale M, Jr (2008) Differential recognition of double-stranded RNA by RIG-I-like receptors in antiviral immunity. J Exp Med 205:1523-1527

34. Taghavi N, Samuel CE (2012) Protein kinase PKR catalytic activity is required for the PKR-dependent activation of mitogen-activated protein kinases and amplification of interferon beta induction following virus infection. Virology 427:208-216

35. Taghavi N, Samuel CE (2013) RNA-dependent protein kinase PKR and the ZDNA binding orthologue PKZ differ in their capacity to mediate initiation factor elF2alpha-dependent inhibition of protein synthesis and virusinduced stress granule formation. Virology 443:48-58

36. Zinzula L, Tramontano E (2013) Strategies of highly pathogenic RNA viruses to block dsRNA detection by RIG-l-like receptors: Hide, mask, hit. Antiviral Res 100:615-635

37. Qin Q, Carroll K, Hastings C, Miller CL (2011) Mammalian orthoreovirus escape from host translational shutoff correlates with stress granule disruption and is independent of elF2alpha phosphorylation and PKR. J Virol 85:8798-8810

38. Holm GH, Zurney J, Tumilasci V, Leveille S, Danthi P, Hiscott J, Sherry B, Dermody TS (2007) Retinoic acid-inducible gene-I and interferon-beta promoter stimulator-1 augment proapoptotic responses following mammalian reovirus infection via interferon regulatory factor-3. J Biol Chem 282:21953-21961

39. Wessel $\varnothing$, Nyman IB, Markussen T, Dahle MK, Rimstad E (2015) Piscine orthoreovirus (PRV) o3 protein binds dsRNA. Virus Res 198:22-29

40. Silverstein SC, Schur PH (1970) Immunofluorescent localization of doublestranded RNA in reovirus-infected cells. Virology 41:564-566

41. Kongtorp RT (2008) Heart and skeletal muscle inflammation (HSMI) in Atlantic salmon, Salmo salar: pathology, pathogenesis and experimental infection. PhD. Norwegian School of Veterinary Science, Oslo

42. Ahmed R, Fields BN (1982) Role of the S4 gene in the establishment of persistent reovirus infection in L cells. Cell 28:605-612

43. Ahmed R, Graham AF (1977) Persistent infections in L cells with temperature-sensitive mutants of reovirus. J Virol 23:250-262

44. Bell TM, Ross MG (1966) Persistent latent infection of human embryonic cells with reovirus type 3. Nature 212:412-414

45. Danis C, Mabrouk T, Garzon S, Lemay G (1993) Establishment of persistent reovirus infection in SC1 cells: absence of protein synthesis inhibition and increased level of double-stranded RNA-activated protein kinase. Virus Res 27:253-265

46. Wetzel JD, Chappell JD, Fogo AB, Dermody TS (1997) Efficiency of viral entry determines the capacity of murine erythroleukemia cells to support persistent infections by mammalian reoviruses. J Virol 71:299-306

47. Parmley RT (1988) Mammals. In: Rowley HM, Ratcliffe NA (ed) Vertebrate blood cells. Cambridge University Press, Cambridge, pp 337-424

48. Dermody TS, Nibert ML, Bassel-Duby R, Fields BN (1990) A o1 region important for hemagglutination by serotype 3 reovirus strains. J Virol 64:5173-5176

49. Weiner HL, Ramig RF, Mustoe TA, Fields BN (1978) Identification of the gene coding for the hemagglutinin of reovirus. Virology 86:581-584

50. Sekhon SS, Beams HW (1969) Fine structure of the developing trout erythrocytes and thrombocytes with special reference to the marginal band and the cytoplasmic organelles. Am J Anat 125:353-373

51. Lane HC, Weaver JW, Benson JA, Nichols HA (1982) Some age related changes of adult rainbow trout, Salmo gairdneri Rich., peripheral erythrocytes separated by velocity sedimentation at unit gravity. J Fish Bio $21: 1-14$

52. Phillips MC, Moyes CD, Tufts BL (2000) The effects of cell ageing on metabolism in rainbow trout (Oncorhynchus mykiss) red blood cells. J Exp Biol 203:1039-1045

53. Piacentini SC, Rohovec JS, Fryer JL (1989) Epizootiology of erythrocytic inclusion body syndrome. J Aquat Anim Health 1:173-179

54. Rodger HD, Richards RH (1998) Observational study of erythrocytic inclusion bodies in farmed Atlantic salmon, Salmo salar L., in the British Isles. J Fish Dis 21:101-111

55. Arakawa CK, Hursh DA, Lannan CN, Rohovec JS, Winton JR (1989) Preliminary characterization of a virus causing infectious anemia among stocks of salmonid fish in the Western United States. In: Ahne W, Kurstak E (ed) Viruses of Lower Vertebrates. Springer, Berlin, pp 442-450
56. Staeheli P, Danielson P, Haller O, Sutcliffe JG (1986) Transcriptional activation of the mouse Mx gene by type I interferon. Mol Cell Biol 6:4770-4774

57. Jorgensen JB, Johansen A, Hegseth MN, Zou J, Robertsen B, Collet B, Secombes CJ (2007) A recombinant CHSE-214 cell line expressing an Mx1 promoter-reporter system responds to both interferon type I and type || from salmonids and represents a versatile tool to study the IFN-system in teleost fish. Fish Shellfish Immunol 23:1294-1303

\section{Submit your next manuscript to BioMed Central and take full advantage of:}

- Convenient online submission

- Thorough peer review

- No space constraints or color figure charges

- Immediate publication on acceptance

- Inclusion in PubMed, CAS, Scopus and Google Scholar

- Research which is freely available for redistribution 